\title{
Efecto de la velocidad de calentamiento sobre las propiedades mecánicas y resistencia a la corrosión de aleaciones de titanio modificadas
}

\author{
Effect of heating rate on the mechanical properties and corrosion resistance of \\ titanium alloy modified
}

\author{
Ma. Mercedes Cely ${ }^{1} \quad$ Grey Castellar O..$^{2^{*}} \quad$ Jhorman Pereira C. ${ }^{3} \quad$ Robert Ángel V. ${ }^{3}$
}

Recibido 13 de junio de 2016, aceptado 6 de noviembre de 2017

Received: June 13, 2016 Accepted: November 6, 2017

\begin{abstract}
RESUMEN
El titanio y sus aleaciones son ampliamente utilizados como materiales biocompatibles, debido a su estabilidad química con el cuerpo humano, una buena relación densidad/resistencia, resistencia a la fatiga, resistencia a la corrosión, entre otros. La capacidad de estos materiales a desarrollar una película pasiva de óxido de manera natural sobre la superficie, lo hace favorable a diferentes aplicaciones, no obstante, la estabilidad de la película es afectada por ambientes agresivos. Los procesos de modificación superficial han sido una alternativa para mejorar la resistencia a la corrosión y algunas propiedades mecánicas. Estas características se deben entre otros a la generación de manera natural de una película de óxido pasiva cuando son expuestos al aire. Esta investigación evaluó el efecto de la velocidad de calentamiento en la oxidación térmica de aleaciones de titanio (Ti6Al4V). Las aleaciones de titanio fueron sometidas a oxidación térmica a 600,700 y $800^{\circ} \mathrm{C}$ con velocidad de calentamiento de 3,4 y $5{ }^{\circ} \mathrm{Cmin}^{-1}$ y enfriamiento isotérmico en horno. La caracterización morfológica de la película de óxido se llevó a cabo mediante Microscopia Electrónica de Barrido y las pruebas de resistencia a la corrosión se realizaron mediante técnicas de polarización potenciodinámica en solución Ringer como electrolito. Los resultados muestran la influencia de la temperatura y la velocidad de calentamiento en el espesor de la película de óxido en un diseño factorial $3^{2}$. Las muestras oxidadas térmicamente a 600 y $800^{\circ} \mathrm{C}$ presentaron mejores resultados respecto a resistencia a la corrosión, además se evidenció un incremento de dureza 2.5 veces mayor en muestras oxidadas a $800^{\circ} \mathrm{C}$ en comparación con el material base.
\end{abstract}

Palabras clave: Aleación Ti6Al4V, oxidación térmica, corrosión, dureza, diseño factorial.

\section{ABSTRACT}

Titanium alloys are widely used as biocompatibles materials, due to their chemical stability inside of the body, a good relationship between density/resistance, fatigue resistance, corrosion resistance and other. The capacity of these materials to develop a naturally passive oxide layer on the surface, makinge them favorable for different applications. However, the stability of the film is affected by aggressive environment. Therefore, the surface modification process has been an alternative to improve the corrosion resistance, and some mechanical properties. This research evaluated the effect of the heating rate on the thermal oxidation of titanium alloys (Ti6Al4V). The method consisted in producing oxide films by thermal oxidation at 600,700 and $800^{\circ} \mathrm{C}$ at different heating rates 3,4 and $5^{\circ} \mathrm{Cmin}^{-1}$ and isothermal cooling in furnace. Morphological characterization was carried out through Scanning Electronic Microscopy, and the corrosion resistance test was carried out by potentiodynamic polarization study using Ringer's solution as an electrolyte. The results showed the influence of the temperature and heating rate on the thickness of the oxide layer in factorial design $3^{2}$. Thermally oxidized

1 Programa de Ingeniería Mecánica, Universidad Autónoma del Caribe, Barranquilla, Colombia. E-mail: mcely@uac.edu.co

2 Programa de Ciencias Básicas, Universidad Autónoma del Caribe, Barranquilla, Colombia. E-mail: grey.castellar@uac.edu.co

3 Ingenieros Mecánicos Programa de Ingeniería Mecánica. Universidad Autónoma del Caribe, Barranquilla, Colombia. E-mail: ing.jhorman@hotmail.com; robertver0306@gmail.com

Autor de correspondencia: grey.castellar@uac.edu.co 
samples at 600 and $800^{\circ} \mathrm{C}$ showed the best results regarding corrosion resistance compared to untreated samples, and the hardness increased 2.5 fold in samples oxidized at $800^{\circ} \mathrm{C}$ compared to the base alloy.

Keywords: Ti6Al4V alloy, thermal oxidation, corrosion, hardness, factorial design.

\section{INTRODUCCIÓN}

El titanio y sus aleaciones poseen buenas propiedades como resistencia a la corrosión, resistencia a altas temperaturas y biocompatibilidad además de su baja densidad lo cual lo hace un material muy utilizado en la industria médica, química [1] así como en la automotriz en la cual se emplea para la construcción de bielas, muelles, sistemas de escape entre otros [2-3] de igual forma es utilizado en la industria aeronáutica para la construcción de partes del fuselaje del avión y componentes de la cámara de compresión como el turbofan [3].

Una característica importante del titanio y sus aleaciones es la generación de manera natural de una película de óxido pasiva a partir de la reacción con el oxígeno, sin embargo esta película presenta baja resistencia al desgaste y en algunos casos, debido a la acción de medios agresivos, puede presentar desprendimiento generando reacciones adversas ya que estas partículas participan como abrasivos de tercer cuerpo que aceleran el desgaste en diferentes interfaces [4-6]. Debido a esto diferentes métodos de modificación superficial, tales como deposición por plasma, nitruración, anodizado, oxidación térmica entre otros, han sido utilizados para mejorar ciertas propiedades de estas aleaciones como el caso de la dureza, la resistencia a la corrosión, la resistencia al desgaste entre otras [7-10].

La oxidación térmica ha sido un método práctico en la generación de espesores de película que favorecen la dureza, la resistencia al desgaste entre otros, Jamesh M. et al. [11] encontraron mediante este método espesores de película del orden de 4 a $20 \mu \mathrm{m}$ de espesor para tiempos de 6 y 14 horas de tratamiento con diferentes velocidades de enfriamiento, así mismo Wang et al, obtuvieron espesores del orden de $7 \mu \mathrm{m}$ para tiempos de 4 horas bajo atmosfera de vapor de agua, mejorando así características de mojabilidad y resistencia a la corrosión [12]. De igual forma algunos autores demostraron como la temperatura y velocidad de enfriamiento son factores que influyen en la formación de la película de óxido, mejorando así propiedades de este material $[9,11,13,14]$.
El objetivo de esta investigación fue determinar el efecto de la oxidación térmica en el crecimiento de la película de óxido con diferentes velocidades de calentamiento, bajo enfriamiento isotérmico mediante la aplicación de un diseño factorial $3^{2}$.

\section{MATERIALES Y MÉTODOS}

Muestras de aleación de titanio -Ti6Al4V- de 3 $\mathrm{mm}$ de espesor $\mathrm{x} 12,7 \mathrm{~mm}$ de diámetro, fueron utilizadas como material base. Antes de la oxidación térmica las muestras fueron desbastadas con lijas de granulometría ASTM desde 320 hasta 1200 y el pulido se realizó con pasta de diamante de $3 \mu \mathrm{m}$, según la norma ASTM E3-01 [15]. Para el análisis metalográfico de las muestras sin tratamiento, se realizó un ataque químico con reactivo kroll's (100 $\mathrm{mL} \mathrm{H} \mathrm{H}_{2} \mathrm{O}+6 \mathrm{~mL} \mathrm{HNO}_{3}+3 \mathrm{~mL}$ HF) durante 30 segundos. La Oxidación térmica fue desarrollada en un horno de resistencias con controlador. Se aplicó un diseño factorial $3^{2}$ con dos factores: la velocidad de calentamiento $\left(3,4\right.$ y $\left.5^{\circ} \mathrm{Cmin}^{-1}\right)$ y la temperatura del proceso $\left(600,700\right.$ y $\left.800{ }^{\circ} \mathrm{C}\right)$, con tres niveles cada uno y tres replicas, la variable respuesta fue la velocidad de corrosión. El tiempo de sostenimiento fue de una hora con enfriamiento isotérmico.

La microestructura se examinó mediante microscopia óptica y el espesor de película obtenido se analizó mediante microscopia electrónica de barrido. Se realizaron pruebas de microdureza con cargas de $200 \mathrm{~g}$ y tiempo de 15 segundos con nueve repeticiones para cada probeta. En las pruebas de resistencia a la corrosión se utilizó una celda con tres electrodos, un electrodo de Ti6Al4V como electrodo de trabajo, un electrodo de $\mathrm{Ag} / \mathrm{AgCl}(\mathrm{KCl}$ $3 \mathrm{M})$ como electrodo de referencia y un electrodo de grafito como contraelectrodo. Las pruebas de polarización potenciodinámicas se realizaron aplicando un barrido de potencial entre $-1,56 \mathrm{~V}$ hasta $0.200 \mathrm{~V}$, con una velocidad de barrido de $1 \mathrm{mVmin}^{-1}$, utilizando solución Ringer (8,4 g de $\mathrm{NaCl}+0,302 \mathrm{~g}$ de $\mathrm{KCl}+0,298 \mathrm{~g}$ de $\mathrm{CaCl}_{2} \cdot 2 \mathrm{H}_{2} \mathrm{O}+0,15 \mathrm{~g}$ de $\mathrm{NaHCO}_{3}$ ) como electrolito. 


\section{RESULTADOS}

\section{Caracterización material base Ti6Al4V}

Las micrografías de la sección transversal y longitudinal de la aleación de titanio Ti6Al4V son mostradas en la figura 1. La aleación posee una matriz $\beta$ contenida en $\alpha$, donde las partes oscuras pertenecen a la estructura $\beta$ $y$ las partes claras corresponden a la estructura $\alpha$. En la sección longitudinal se observan granos alargados debido a la deformación a la cual es sometido el material para su proceso de fabricación mientras que en la sección transversal se observan granos uniformes y equiaxiales.
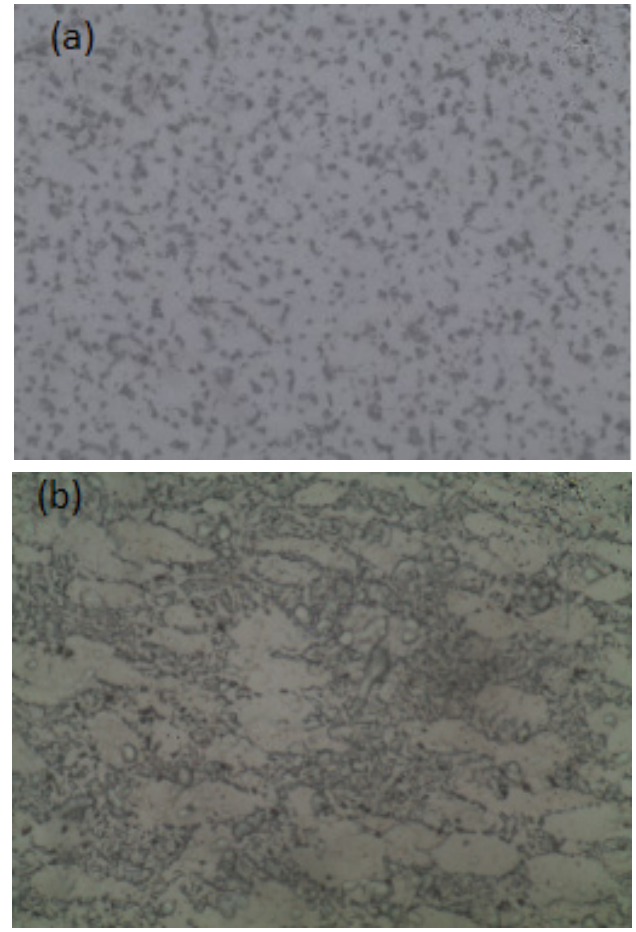

Figura 1. Micrografías de la aleación Ti6Al4V a 100x. a) Sección transversal, b) sección longitudinal.

La Tabla 1 muestra los valores de dureza de la aleación Ti6Al4V sometida a tratamiento térmico. Las probetas con tratamiento a $600^{\circ} \mathrm{C}$ incrementaron en un $5 \%$ su dureza, mientras que a $700^{\circ} \mathrm{C}$ presentaron un incremento alrededor del 30\% en comparación con el material base. Es importante resaltar el aumento de dureza (alrededor del 58\%) de las probetas sometidas a tratamiento de $800^{\circ} \mathrm{C}$, lo anterior debido a la formación de una película de óxido compuesta por diferentes tipos de óxidos como anatasa, rutilo, $\mathrm{TiO}_{3}$ entre otros, los cuales presentan características de dureza y resistencia importantes, como fue evidenciado en estudios previos [16-19]. Por otra parte Pinilla y otros obtuvieron valores similares de dureza para muestras tratadas a $800^{\circ} \mathrm{C}$ con una velocidad de calentamiento de $5^{\circ} \mathrm{Cmin}^{-1}$, sin embargo, se produjo un desprendimiento de la capa de óxido generado por la diferencia de los coeficientes de expansión térmica entre esta y el substrato por enfriamiento más acelerado [16], [20].

Respecto a las velocidades de calentamiento, las mayores durezas se encontraron a velocidades de $3^{\circ} \mathrm{Cmin}^{-1}$ en todos los casos, con una diferencia más significativa para temperaturas de tratamiento de $800^{\circ} \mathrm{C}$, cuyas muestras no evidenciaron desprendimiento de la película de óxido, posiblemente por el efecto isotérmico en el proceso de enfriamiento de las probetas.

Tabla 1. Microdureza Vickers de la aleación Ti6Al4V a temperaturas de 600,700 y $800^{\circ} \mathrm{C}$ de tratamiento.

\begin{tabular}{|c|c|c|c|}
\hline $\begin{array}{l}\text { Temperatura } \\
\text { de tratamiento } \\
\left({ }^{\circ} \mathrm{C}\right)\end{array}$ & $\begin{array}{l}\text { Velocidad de } \\
\text { calentamiento } \\
\left({ }^{\circ} \mathrm{Cmin}^{-1}\right)\end{array}$ & $\begin{array}{l}\text { Código de } \\
\text { designación }\end{array}$ & $\begin{array}{l}\text { Micro-dureza } \\
(\mathrm{HV})_{0.2} \pm \text { D.S }\end{array}$ \\
\hline $\begin{array}{c}\text { Sin } \\
\text { tratamiento }\end{array}$ & & $\begin{array}{l}\text { Material } \\
\text { base }\end{array}$ & $330.5 \pm 4.3$ \\
\hline 600 & 3 & P600-3C & $346.6 \pm 4.7$ \\
\hline 600 & 4 & P600-4C & $349.0 \pm 4.7$ \\
\hline 600 & 5 & P600-5C & $346.6 \pm 0.9$ \\
\hline 700 & 3 & P700-3C & $480.6 \pm 11.7$ \\
\hline 700 & 4 & P700-4C & $466.2 \pm 5.6$ \\
\hline 700 & 5 & P700-5C & $465.8 \pm 29.6$ \\
\hline 800 & 3 & P800-3C & $862.2 \pm 46.7$ \\
\hline 800 & 4 & P800-4C & $749.2 \pm 26.1$ \\
\hline 800 & 5 & P800-5C & $739.3 \pm 17.3$ \\
\hline
\end{tabular}

A mayor temperatura de tratamiento se encontraron mayores valores de dureza, sin embargo, la desviación estándar se incrementó, esto debido al crecimiento no uniforme de cristales en la capa de óxido, lo que dificultó la medición de dichos valores siendo influyentes en su comportamiento, para este estudio solo se tuvieron en cuenta el espesor de película para muestras sometidas a tratamiento de $800^{\circ} \mathrm{C}$.

En la Figura 2 se presenta la vista transversal de la película de óxido formada en las muestras de la aleación Ti6Al4V sometidas a oxidación térmica a la condición de $800^{\circ} \mathrm{C}$ con velocidades de calentamiento de 3,4 y $5^{\circ} \mathrm{Cmin}^{-1}$ y enfriamiento isotérmico en el horno. 


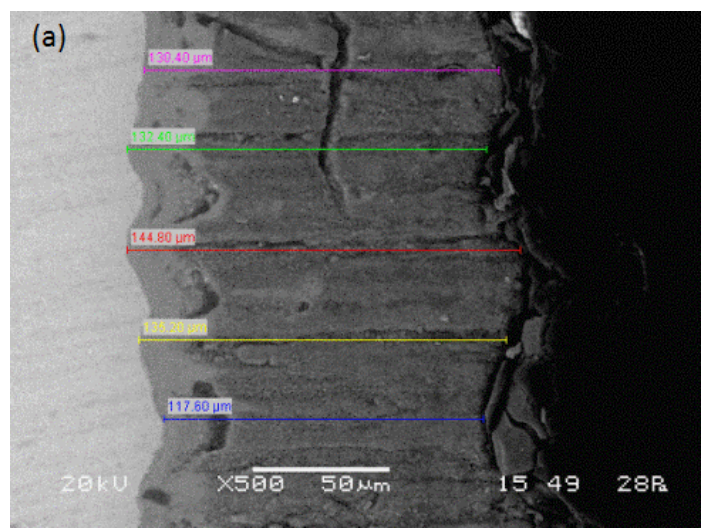

(b)

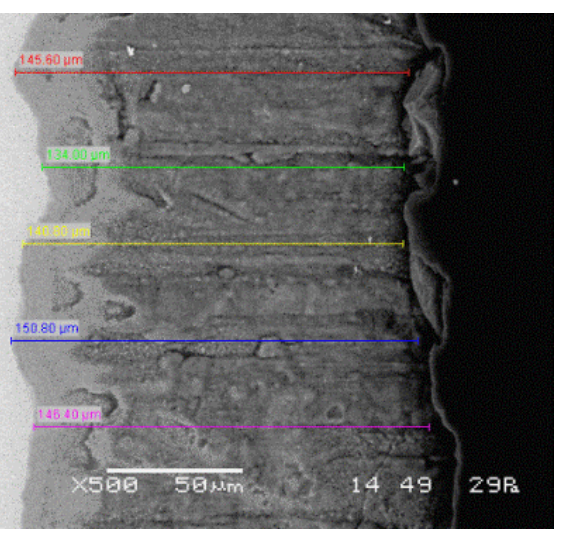

(c)

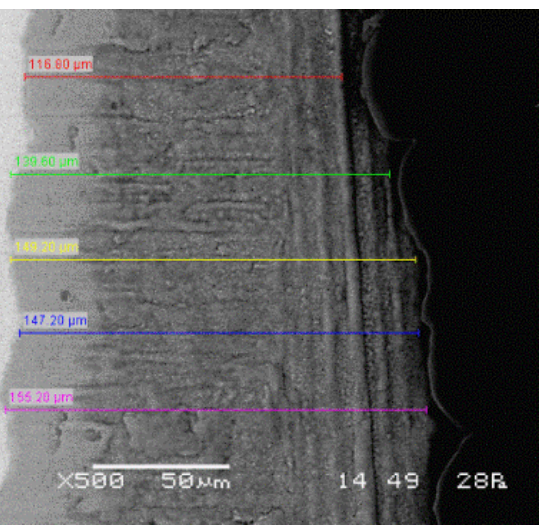

Figura 2. Corte transversal de la película de óxido obtenida a $800^{\circ} \mathrm{C}$ con velocidades de (a) $3^{\circ} \mathrm{Cmin}^{-1}$, (b) $4^{\circ} \mathrm{Cmin}^{-1}$, (c) $5^{\circ} \mathrm{Cmin}^{-1}$.

El cambio de la morfología de la película de óxido en función de la temperatura de oxidación sugiere que el mecanismo de formación de la película de óxido se debe a la gran solubilidad que presenta el oxígeno con el titanio en la formación de óxidos [17], [8], [20-21].

En la Figura 3 se pueden observar los espesores de capa de óxido y la desviación estándar de los valores en cada muestra después de la oxidación térmica.

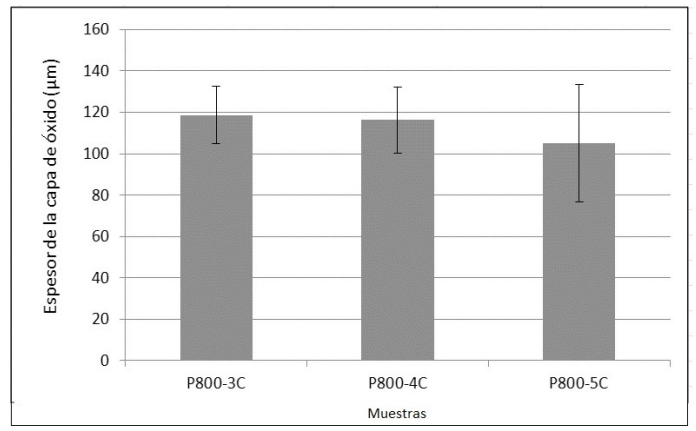

Figura 3. Espesores de película de óxidos de la aleación Ti6A14V después de la oxidación térmica.

La Figura 3 muestra que las probetas oxidadas a $800^{\circ} \mathrm{C}$ con distintas velocidades de calentamiento generaron películas de óxido del orden de $110 \pm 10 \mathrm{HV}_{200}$, con tiempo de sostenimiento de 1 hora siendo este un aspecto importante en comparación a resultados encontrados en la literatura en la cual se muestra que con un tiempo de sostenimiento de 4 y 6 horas y temperatura de calentamiento de $800^{\circ} \mathrm{C}$ se obtuvieron espesores de 34,9 y $20 \mu \mathrm{m}$ respectivamente, con enfriamiento en el horno [17],[11].

Se registra que en el proceso de oxidación térmica a medida que aumenta la temperatura hay un incremento en la dureza superficial y un crecimiento en la capa de óxido de la aleación, como lo evidenció H. Guleryuz et al [22].

La Figura 4 muestra las curvas de polarización para la aleación Ti6Al4V tratada a 600,700 y $800^{\circ} \mathrm{C}$, bajo 3 velocidades de calentamiento. Se puede observar como las ramas anódicas muestran una transición activa -pasiva en todos los casos.

Las muestras oxidadas térmicamente a $600^{\circ} \mathrm{C}$ bajo las tres condiciones de tratamiento $\left(3,4\right.$ y $\left.5^{\circ} \mathrm{Cmin}-1\right)$ presentan valores de potencial de corrosión (Ecorr) más nobles con respecto al material base y a las condiciones de 700 y $800^{\circ} \mathrm{C}$. Además, se observó una relación entre la velocidad de calentamiento y el potencial de corrosión en las muestras tratadas a 700 y $800^{\circ} \mathrm{C}$. Los valores de Ecorr de las muestras oxidadas a $700^{\circ} \mathrm{C}$ disminuyeron con el aumento de la velocidad de calentamiento; caso contrario el de las muestras oxidadas a $800^{\circ} \mathrm{C}$, donde a mayor velocidad de calentamiento, los valores de Ecorr fueron más nobles.

La Tabla 2 presenta los valores de potencial de corrosión y densidad de corriente de corrosión para las diferentes 
velocidades de calentamiento. Allí se pudo observar que las probetas tratadas a $600^{\circ} \mathrm{C}$ presentaron valores menores de densidad de corriente de corrosión (Icorr) comparado con las otras muestras, a excepción de la muestras oxidadas a $800^{\circ} \mathrm{C}$ y $5^{\circ} \mathrm{Cmin}^{-1}$ cuyos valores fueron similares, lo anterior debido a la formación de rutilo en la capa de óxido, cuyas propiedades de resistencia a la corrosión son demostradas en la literatura [17],[19].

Las muestras tratadas a $600^{\circ} \mathrm{C}$ presentaron valores más nobles de Ecorr y valores menores de Icorr, indicando un carácter protector, presentando así un mejor comportamiento ante procesos de corrosión.
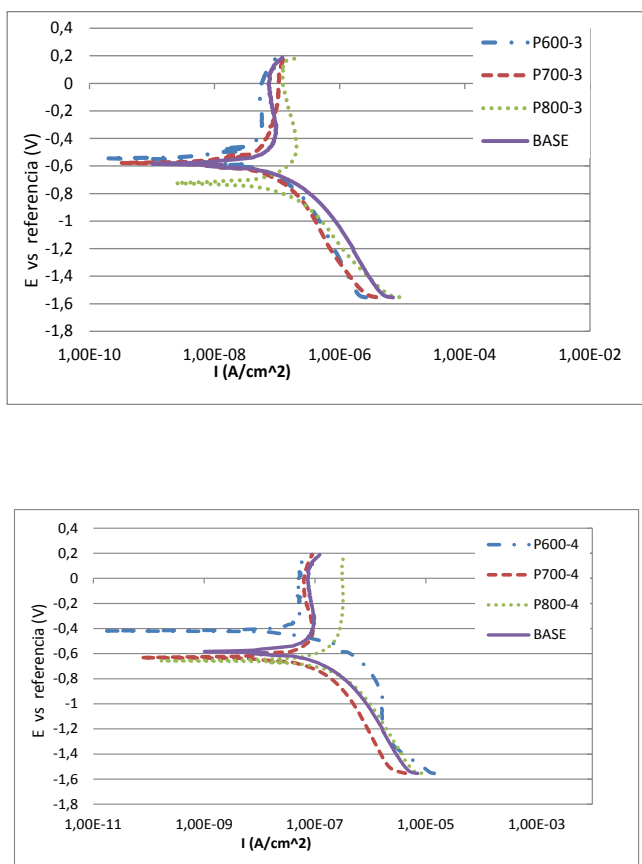

(b)

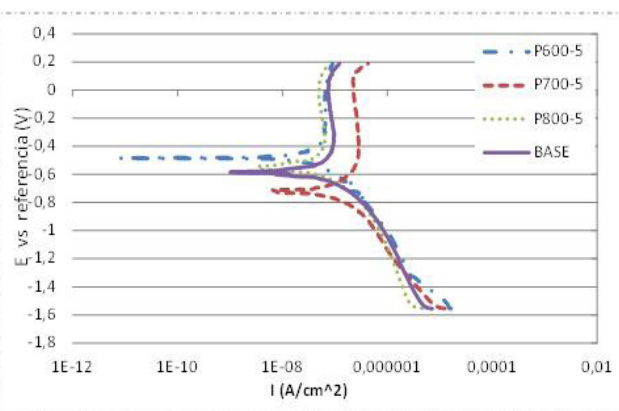

Figura 4. Curvas de polarización para la aleación tratada a 600,700 y $800^{\circ} \mathrm{C}$. Velocidad de calentamiento a) $0,3^{\circ} \mathrm{Cmin}^{-1}$, b) $0,4^{\circ} \mathrm{Cmin}^{-1}$, y c) $0,5^{\circ} \mathrm{Cmin}^{-1}$.
Tabla 2. Valores de $\mathrm{E}_{\text {corr }}$ e $\mathrm{i}_{\text {corr }}$ en la aleación Ti6Al4V.

\begin{tabular}{|c|c|c|c|c|}
\hline 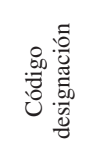 & 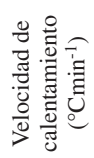 & 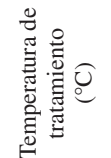 & 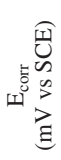 & • \\
\hline $\begin{array}{l}\text { Material } \\
\text { base }\end{array}$ & & $\begin{array}{l}\text { Sin } \\
\text { tratamiento }\end{array}$ & -580 & 7.83 \\
\hline P600-3C & 3 & 600 & -540 & 5,34 \\
\hline P600-4C & 4 & 600 & -410 & 4,92 \\
\hline P600-5C & 5 & 600 & -480 & 7,18 \\
\hline P700-3C & 3 & 700 & -570 & 10,8 \\
\hline P700-4C & 4 & 700 & -630 & 6,35 \\
\hline P700-5C & 5 & 700 & -680 & 27,6 \\
\hline P800-3C & 3 & 800 & -720 & 12,9 \\
\hline P800-4C & 4 & 800 & -650 & 31,2 \\
\hline P800-5C & 5 & 800 & -560 & 4,91 \\
\hline
\end{tabular}

La Tabla 3, muestra el cuadro de análisis de varianza. El cuadro ANOVA descompone la variabilidad del potencial de corrosión o Ecorr en contribuciones debidas a varios factores. Puesto que se ha escogido la suma de cuadrados Tipo III (por omisión), la contribución de cada factor se mide eliminando los efectos de los demás factores. Los valores-P prueban la significancia estadística de cada uno de los factores. Se reconoce una diferencia significativa cuando valor de $P$ es 0,05 o menor; puesto que un valor-P es menor que 0,05 para el caso del factor temperatura, este factor tiene un efecto estadísticamente significativo sobre el potencial de corrosión o Ecorrosión con un 95,0\% de nivel de confianza.

Para estudiar el efecto de potencial de corrosión fue utilizado un diseño factorial multinivel con dos factores, utilizando la herramienta de estadística Statgraphic Century, este procedimiento ejecuta un análisis de varianza de varios factores para el potencial de corrosión ( $\left.\mathrm{E}_{\text {corr }}\right)$.

Todas las razones-F se basan en el cuadrado medio del error residual.

Se presentó una mayor diferencia a velocidades de calentamiento de 4 y $5^{\circ} \mathrm{Cmin}^{-1}$, generándose diferencias significativas entre 600 y $700^{\circ} \mathrm{C}$. Las muestras sometidas a tratamientos de $600^{\circ} \mathrm{C}$ presentaron un buen comportamiento a la corrosión, sin embargo, se debe resaltar que muestras a $800^{\circ} \mathrm{C}$ presentaron potenciales de corrosión muy similares al material base, que generaron una película de óxido protectora con durezas casi 2,5 veces mayor que la del material base. 
La Figura 5 muestra un diagrama de dispersión del potencial de corrosión contra la temperatura; allí se observa una leve tendencia de potenciales más negativos con el incremento de temperatura, siendo más significativo para $700^{\circ} \mathrm{C}$.

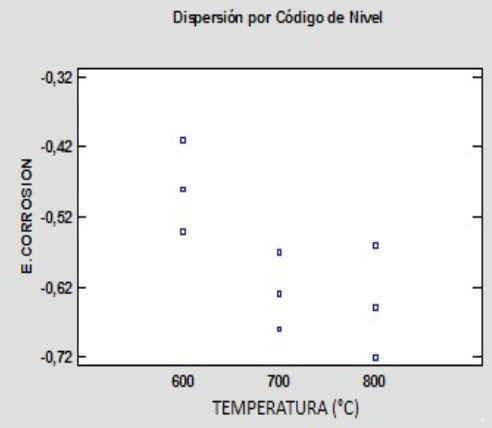

Figura 5. Diagrama de dispersión por código de nivel del Ecorrosión contra la temperatura.

Las pruebas-F en la tabla ANOVA permiten identificar los factores significativos. Para cada factor significativo, las Pruebas de Rangos Múltiples nos indican cuales medias son significativamente diferentes de otras.
Tabla 4. Pruebas de Múltiple Rangos para Ecorrosión por temperatura.

Método: 95,0 porcentaje LSD

\begin{tabular}{|l|c|l|l|l|}
\hline$T\left({ }^{\circ} \mathrm{C}\right)$ & Casos & Media LS & Sigma LS & $\begin{array}{l}\text { Grupos } \\
\text { Homogéneos }\end{array}$ \\
\hline 700 & 6 & $-0,653333$ & 0,025074 & $\mathrm{X}$ \\
\hline 800 & 6 & $-0,638333$ & 0,025074 & $\mathrm{X}$ \\
\hline 600 & 6 & $-0,503333$ & 0,025074 & $\mathrm{X}$ \\
\hline
\end{tabular}

\begin{tabular}{|l|c|l|l|}
\hline Contraste & Sig. & Diferencia & +/- Limites \\
\hline $600-700$ & $*$ & 0,15 & 0,0802161 \\
\hline $600-800$ & $*$ & 0,135 & 0,0802161 \\
\hline $700-800$ & & $-0,015$ & 0,0802161 \\
\hline
\end{tabular}

* indica una diferencia significativa.

No existen diferencias estadísticamente significativas entre aquellos niveles que compartan una misma columna de X's, lo que indica que las diferencias de los tratamientos son significativas con respecto a la temperatura de $600^{\circ} \mathrm{C}$. El método de Fisher empleado actualmente para discriminar entre las medias es un método donde hay un riesgo del 5,0\% al decir que cada par de medias es significativamente diferente, cuando la diferencia real es igual a 0 .

Tabla 3. Análisis de varianza para Ecorrosión suma de cuadrados Tipo III.

\begin{tabular}{|l|c|c|c|c|c|}
\hline \multicolumn{1}{|c|}{ Fuente } & Suma de cuadrados & Gl & Cuadrado medio & Razón-F & Valor-P \\
\hline EFECTOS PRINCIPALES & & & & & \\
\hline A:Temperatura & 0,0819 & 2 & 0,04095 & 10,86 & 0,0040 \\
\hline B:Velocidad de calentamiento & 0,0026 & 2 & 0,00131 & 0,35 & 0,7145 \\
\hline INTERACCIONES & & & & & \\
\hline AB & 0,0287 & 4 & 0,00719 & 1,91 & 0,1936 \\
\hline RESIDUOS & 0,0339 & 9 & 0,00377 & & \\
\hline TOTAL (CORREGIDO) & 0,1472 & 17 & & & \\
\hline
\end{tabular}

La Tabla 4 muestra las pruebas de rangos por el método de diferencia mínima significativa (LSD) de Fisher. Estos datos buscan una comparación múltiple para determinar cuáles medias son significativamente diferentes de otras.

La mitad inferior de la salida muestra las diferencias estimadas entre cada par de medias. El asterisco que se encuentra al lado de los 2 pares indica que estos pares muestran diferencias estadísticamente significativas con un nivel del 95,0\% de confianza. En la parte superior de la página, se han identificado 2 grupos homogéneos según la alineación de las $\mathrm{X}$ 's en columna.

\section{CONCLUSIONES}

La morfología superficial en las muestras oxidadas a $800^{\circ} \mathrm{C}$ presentó un crecimiento del óxido, con granos más pequeños en las muestras con velocidad de calentamiento de $5^{\circ} \mathrm{Cmin}^{-1}$.

La dureza aumentó 2,5 veces más en comparación con la aleación no tratada, cuando el tratamiento aplicado se lleva hasta los $800^{\circ} \mathrm{C}$, pasando de 316 $\pm 7,0$ a $802 \pm 46,45 \mathrm{HV}_{0.2}$.

Los valores de potencial de corrosión ( $\left.\mathrm{E}_{\mathrm{corr}}\right)$ que presentaron mejores condiciones se encontraron 
a temperaturas de $600^{\circ} \mathrm{C}$ para las 3 velocidades de calentamiento, sin embargo, no hay diferencias significativas respecto a estos tratamientos.

El factor "temperatura de tratamiento" presentó diferencia significativa entre los tratamientos, lo que se evidencia no solo en los espesores de película obtenidos, sino además, en su comportamiento respecto a la resistencia a la corrosión [23].

\section{AGRADECIMIENTOS}

Los autores dan su agradecimiento a la Universidad Autónoma del Caribe por la financiación del proyecto.

\section{REFERENCIAS}

[1] D. Rodríguez, Obtención de capas de nitruro de titanio por tratamientos termoquímicos en Titanio y Ti6Al4V y caracterización de las propiedades para aplicaciones biomédicas, Universidad Politecnica de Cataluña, 1999.

[2] M. Duraiselvam, a. Valarmathi, S.M. Shariff, G. Padmanabham, Laser surface nitrided Ti-6Al-4V for light weight automobile disk brake rotor application, Wear. 309 (2014) 269-274. doi:10.1016/j.wear.2013.11.025.

[3] A. Mouritz, Introducción a los Materiales Aeroespaciales, Woodhead Publ. Ltd. (ISBN 9871 (2012).

[4] Z. Abdolldhi, A.A.M. Ziaee, A. Afshar, Investigation of Titanium Oxide Layer in Thermal-Electrochemical Anodizing of Ti6Al4V Alloy, Biomol. Eng. (2009) 44-48.

[5] S. Roessler, R. Zimmermann, D. Scharnweber, C. Werner, H. Worch, Characterization of oxide layers on Ti6Al4V and titanium by streaming potential and streaming current measurements, Colloids Surfaces B Biointerfaces. 26 (2002) 387-395. doi:10.1016/S0927-7765(02)00025-5.

[6] C. Marino, E.M. De Oliveira, R.C. Rocha-, S.R. Biaggio, On the stability of thin-anodic-oxide films of titanium in acid phosphoric media, Corros. Sci. 43 (2001) 1465-1476.

[7] S. Kumar,T.S.N.S. Narayanan, S. Ganesh Sundara Raman, S.K. Seshadri, Surface modification of CP-Ti to improve the fretting-corrosion resistance: Thermal oxidation vs. anodizing, Mater. Sci. Eng. C. 30 (2010) 921-927. doi:10.1016/j. msec.2010.03.024.

[8] X. Liu, P.K. Chu, C. Ding, Surface modification of titanium, titanium alloys, and related materials for biomedical applications, Mater. Sci. Eng. R Reports. 47 (2004) 49-121. doi:10.1016/j. mser.2004.11.001.

[9] M.T. Mohammed,Z.A. Khan, A.N. Siddiquee, Surface Modifications of Titanium Materials for developing Corrosion Behavior in Human Body Environment: A Review, Procedia Mater. Sci. 6(2014) 1610-1618. doi:10.1016/j. mspro.2014.07.144.

[10] K. Das, S. Bose, A. Bandyopadhyay, Surface modifications and cell-materials interactions with anodized Ti., Acta Biomater. 3 (2007) 573-85. doi:10.1016/j.actbio.2006.12.003.

[11] M. Jamesh, T.S.N. Sankara Narayanan, P.K. Chu, Thermal oxidation of titanium: Evaluation of corrosion resistance as a function of cooling rate, Mater. Chem. Phys. 138 (2013) 565-572. doi:10.1016/j.matchemphys.2012.12.020.

[12] S. Wang, Y. Liu, C. Zhang, Z. Liao, W. Liu, The improvement of wettability, biotribological behavior and corrosion resistance of titanium alloy pretreated by thermal oxidation, Tribol. Int. 79 (2014) 174-182. doi:10.1016/j. triboint.2014.06.008.

[13] S. Wang, Z. Liao, Y. Liu, W. Liu, Influence of thermal oxidation temperature on the microstructural and tribological behavior of Ti6Al4V alloy, Surf. Coatings Technol. 240 (2014) 470-477. doi:10.1016/j.surfcoat.2014.01.004.

[14] M.Wen, C. Wen, P.Hodgson, Y.Li, Improvement of the biomedical properties of titanium using SMAT and thermal oxidation, Colloids Surfaces B Biointerfaces. (2013). doi:10.1016/j. colsurfb.2013.10.039.

[15] ASTM Norma, E3-11 Standard Guide for Preparation of Metallographic Specimens 1,ASTM Copyright. (2011) 1-12. doi:10.1520/E0003-11.2.

[16] D. Sivaramakrishna, Y. Brama,Y. Sun, Thick rutile layer on titanium for tribological applications, Tribol. Int. 40 (2007) 329-334. doi:10.1016/j. triboint.2005.08.004.

[17] S. Wang, Z. Liao, Y.Liu and W. Liu, Influence of thermal oxidation duration on the microstructure and fretting wear behavior of Ti6A14V alloy, Mater. Chem. Phys.159 (2015) 139-151. doi: 10.1016/j.matchemphys.2015.03.063.

[18] T.M. Lee, Effect of passivation and surface modification on the dissolution behavior and nano-surface characteristics of Ti-6Al4V in Hank/EDTA solution., J. Mater. Sci. Mater. Med. 17 (2006) 15-27. doi:10.1007/ s10856-006-6325-3. 
[19] L.-C. Chuang, C.-H. Luo, S. Yang, The structure and mechanical properties of thick rutile-TiO2 films using different coating treatments, Appl. Surf. Sci. 258 (2011) 297-303. doi:10.1016/j. apsusc.2011.08.055.

[20] I. Vaquila,L.I.Vergara, M.C.G.Passeggi, R. a. Vidal, J. Ferrón, Chemical reactions at surfaces: Titanium oxidation, Surf. Coatings Technol. 122 (1999) 67-71. doi:10.1016/S0257-8972(99)00420-X.

[21] O. Pinilla, A. Siado, Caracterizacion Microestructural de la Aleacion de Titanio
Ti6A14V Oxidada Térmicamente., Universidad Autónoma del Caribe, 2014.

[22] H. Guleryuz, H. Cimenoglu, Effect of thermal oxidation on corrosion and corrosion-wear behaviour of a Ti-6Al-4V alloy. Biomaterials 25 (2004) 3325, (n.d.).

[23] Y. Liu, D.Z. Yang, S.Y. He, W.L. Wu, Microstructure developed in the surface layer of Ti-6Al-4V alloy after sliding wear in vacuum, Mater. Charact. 50 (2003) 275-279. doi:10.1016/S1044-5803(03)00125-6. 